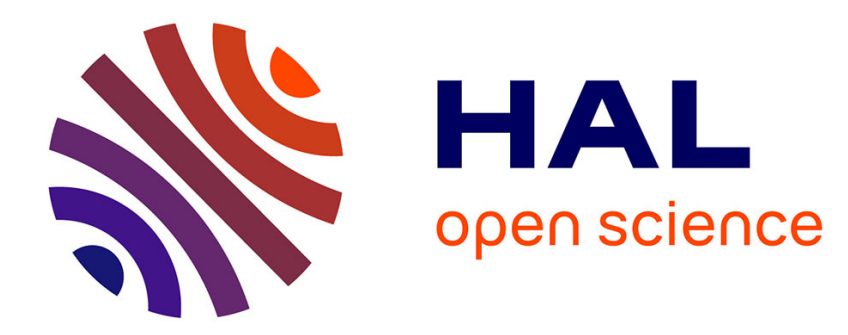

\title{
Electro-optic measurement of the order parameter of $\mathrm{NH} 4 \mathrm{Cl}$
}

\author{
J. P. Pique, G. Dolino, M. Vallade
}

\section{To cite this version:}

J. P. Pique, G. Dolino, M. Vallade. Electro-optic measurement of the order parameter of NH4Cl. Journal de Physique, 1978, 39 (9), pp.1003-1013. 10.1051/jphys:019780039090100300 . jpa-00208829

\section{HAL Id: jpa-00208829 https://hal.science/jpa-00208829}

Submitted on 1 Jan 1978

HAL is a multi-disciplinary open access archive for the deposit and dissemination of scientific research documents, whether they are published or not. The documents may come from teaching and research institutions in France or abroad, or from public or private research centers.
L'archive ouverte pluridisciplinaire HAL, est destinée au dépôt et à la diffusion de documents scientifiques de niveau recherche, publiés ou non, émanant des établissements d'enseignement et de recherche français ou étrangers, des laboratoires publics ou privés. 


\title{
ELECTRO-OPTIC MEASUREMENT OF THE ORDER PARAMETER OF $\mathrm{NH}_{4} \mathrm{Cl}$
}

\author{
J. P. PIQUE, G. DOLINO and M. VALLADE \\ Laboratoire de Spectrométrie Physique $\left(^{*}\right)$ \\ Université Scientifique et Médicale de Grenoble, B.P. no 53, 38041 Grenoble Cedex, France
}

(Reçu le 10 avril 1978, accepté le 31 mai 1978)

\begin{abstract}
Résumé. - Nous présentons ici des mesures de la variation avec la température de l'effet électrooptique du chlorure d'ammonium dans sa phase basse température et nous montrons comment elles sont reliées au paramètre d'ordre de cette transition. Ces mesures sont rendues difficiles par la présence d'une biréfringence parasite de déformation et par l'existence de domaines où l'effet électrooptique change de signe. Nos résultats sont comparés à des mesures antérieures du paramètre d'ordre par effet piézoélectrique et par génération de seconde harmonique. Nous discutons l'interprétation de ces mesures utilisant la théorie de Landau ou une loi de puissance avec des exposants critiques et la relation avec l'existence d'un point multicritique sous haute pression.

Une queue exponentielle du paramètre d'ordre s'étendant sur plusieurs degrés dans la phase haute température est systématiquement observée dans toutes les expériences. L'origine de cet ordre résiduel est probablement reliée aux contraintes produites par des défauts cristallographiques.
\end{abstract}

\begin{abstract}
We present here measurements of the temperature variation of the electro-optic effect in the low temperature phase of $\mathrm{NH}_{4} \mathrm{Cl}$ and we show how they are related to the order parameter of the order-disorder transition. These measurements are made difficult by the presence of parasitic strain birefringence, and by the existence of domains which produce a change of sign of the electro-optic effect. The results are compared with previous measurements of the order parameter by the piezoelectric effect and second harmonic generation, and their interpretation, following the Landau theory or power law with critical exponents, is discussed in relation to the existence of a multicritical point under high pressure.

An exponential tail of the order parameter, extending over several degrees in the high temperature phase is systematically found in all these experiments. The origin of this residual order is probably related to the stresses produces by crystalline defects.
\end{abstract}

1. Introduction. - Ammonium chloride undergoes an order-disorder phase transition near a temperature $T_{\mathrm{c}}=242 \mathrm{~K}$ [1]. In the high temperature phase the $\mathrm{NH}_{4}^{+}$tetraedra are oriented at random between two positions, whereas they take parallel orientations in the low temperature phase. $\mathrm{NH}_{4} \mathrm{Cl}$ is of particular interest since the mechanism of the transition is very simple and provides a physical realisation of a compressible Ising model [2]. As shown by Huller [3] the ordering of $\mathrm{NH}_{4}^{+}$in ammonium halides results from a competition between a direct octupole-octupole interaction, favoring parallel ordering, and an indirect octupole-dipole-octupole interaction via the anion, favoring antiparallel orientation. This antiparallel

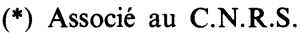

orientation exists in $\mathrm{NH}_{4} \mathrm{Br}$ [4] and in mixed crystals such as $\mathrm{NH}_{4} \mathrm{Br}_{x} \mathrm{Cl}_{1-x}[5,6]$. A tendency towards an antiparallel order in $\mathrm{NH}_{4} \mathrm{Cl}$ is shown in the diffuse $\mathrm{X}$-ray scattering produced at room temperature by thermal fluctuations [7].

It now seems well established that the transition which is of first order and so discontinuous at room temperature becomes continuous under high pressure $(1,5 \mathrm{kbar})[8,9]$ but the exact nature of this multicritical point is still subject to discussion [10]. The neutron scattering experiment by Yelon et al. [1] on $\mathrm{ND}_{4} \mathrm{Cl}$ was interpreted as showing the existence of a tricritical point but the low value $(\beta=0.153)$ of the critical exponent for the temperature variation of the order parameter has led some authors to think that this is a multicritical point of higher order $[10,11]$ Therefore it seems interesting to make new accurate 
measurements of a quantity directly related to the order parameter $\eta$. In $\mathrm{NH}_{4} \mathrm{Cl}$ the high temperature phase belongs to the octahedral group $\mathrm{O}_{h}$ and is centrosymmetric while the low temperature phase belongs to a non centrosymmetric, non polar tetrahedral group $\left(\mathrm{T}_{\mathrm{d}}\right)$.

Macroscopic physical quantities possessing the same symmetry transformation properties as the order parameter $\eta\left(\mathrm{A}_{2}\right.$ representation of the $\mathrm{O}_{h}$ group) are described by a 3rd (or higher order) rank tensor [12]. Physical quantities with such symmetry properties are the piezoelectric effect, the electro-optic effect and second harmonic generation of light.

Unfortunately the measurements of the order parameter by these techniques are made difficult by the existence of a domain structure [13]. In fact $\eta=\left(n_{+}-n_{-}\right) /\left(n_{+}+n_{-}\right)$where $n_{+}$and $n_{-}$are the number of tetraedra $\mathrm{NH}_{4}^{+}$possessing each of the two possible orientations. $\eta$ can take two opposite values below $T_{\mathrm{c}}$ and this leads to a domain structure. All the physical tensors having the same symmetry as $\eta$ change their sign in opposite domains, and their mean value on the sample may be much less than their local value inside one domain. Usually a crystal may be made single domain by applying the field conjugated to the order parameter. But, in the case of $\mathrm{NH}_{4} \mathrm{Cl}$ this field must be a third order tensor, which is not experimentally available. Nevertheless products of stresses $\sigma_{i j}$ and electric field $E_{i}$ such as $\sigma_{x y} E_{z}, E_{x} E_{y} E_{z} \ldots$ are linearly coupled to $\eta$. These quantities cannot be considered as the true conjugated parameter since they break the cubic symmetry of the low temperature phase. Nevertheless they lift the degeneracy between $+\eta$ and $-\eta$ and consequently can influence the domain structure [14]. Furthermore a susceptibility such as $\partial \eta / \partial\left(\sigma_{x y} E_{z}\right)$ is expected to reflect the fluctuations $\left\langle\eta^{2}\right\rangle$ of the order parameter and to increase near the transition. Among these properties the piezoelectric effect has recently been investigated at atmospheric pressure by Mohler and Pitka [15] and by Bruins and Garland [11]. Mohler and Pitka realized their measurements on a single domain sample obtained by the application of an electric field and of a stress. They have interpreted their result with a power law $\eta \sim\left(T^{*}-T\right)^{\beta}$ where the critical exponent $\beta=0.33$; Bruins and Garland investigated polydomain samples and found $\beta=0.134$. They have also noted the persistance of long range order several degrees above the transition temperature. This puzzling feature has also been observed by Freund and Kopf [16] and by Steinbrener and Jahn [17] in second harmonic measurements. The temperature dependence of the electro-optic effect has been reported recently by Konak [18, 19] and by the present authors [20]. In this paper we give a complete description of our results at atmospheric pressure.

In part 2 we describe the properties of the electrooptic effect in a cubic crystal such as $\mathrm{NH}_{4} \mathrm{Cl}$ and consider the parasitic effects of internal strains. In part 3 we describe the experimental set-up and the results in samples with various kinds of domain structures. Detailed comparisons of our results with previous order parameter measurements and theoretical formulae are discussed in part 4 in connection with the nature of the multicritical point. Possible mechanisms for the persistance of some order above the transition temperature are also presented.

2. Electro-optic effect in $\mathbf{N H}_{4} \mathbf{C l}$. - As the crystal belongs to cubic groups in the two phases, it is optically isotropic in the absence of internal stresses and of external fields. With the application of an electric field $E_{k}$ the coefficients $B_{i j}$ of the optical index ellipsoid are given by [21] :

$$
B_{i j}=\frac{1}{n^{2}}+r_{i j k}^{\mathrm{e}} E_{k}+R_{i j k l} E_{k} E_{l}+\cdots
$$

where $n$ is the refractive index in the absence of a field.

The term associated to $r_{i j k}^{\mathrm{e}}$ in equation (1) describes the linear electro-optic (or Pockels effect), which vanishes in the high temperature phase. In the low temperature phase the only non zero coefficients are $r_{x y z}^{\mathrm{e}}=r_{z x y}^{\mathrm{e}}=r_{y z x}^{\mathrm{e}}=r_{41}^{\mathrm{e}}$ in the ordinary cubic axis using Voigt notation. The last term in equation (1) represents the quadratic electro-optical (or Kerr effect) which exists in the two phases. As the crystal also becomes piezoelectric below $T_{\mathrm{c}}$, in addition to the direct electro-optic effect given by equation (1) for a clamped crystal, there is an indirect effect produced by the piezoelectric deformation in a free crystal, so that the total effect is given by :

$$
r_{41}=r_{41}^{\mathrm{e}}+p_{44} d_{14}=r_{41}^{\mathrm{e}}+r_{41}^{\mathrm{i}}
$$

where the piezoelectric constant relating strains to the electric field is $d_{14} \sim 10^{-9} \mathrm{~cm} / \mathrm{V}$ [15] and the photoelastic constant relating the refractive indices to the strain is $p_{44}=2.5 \times 10^{-2}$ [22]. Then the indirect part $r_{41}^{\mathrm{i}}=2.5 \times 10^{-11} \mathrm{~cm} / \mathrm{V}$ while the total effect is $r_{41} \gtrsim 2 \times 10^{-10} \mathrm{~cm} / \mathrm{V}$.

The indirect effect is smaller than the direct effect, but not negligible. However $p_{44}$ is expected to have a small temperature variation while $d_{14}$ and $r_{41}^{\mathrm{e}}$ are expected to have similar temperature variations, as they are linearly related to $\eta$ (in a first approximation). So in the following we shall consider only the total effect related to $r_{41}$.

For a laser beam propagating along the $\langle 110\rangle$ direction and an electric field $E$ along $\langle 001\rangle$ the electro-optical retardation angle $\phi$ is given by :

$\phi=\frac{2 \pi l}{\lambda} \Delta n=+\frac{2 \pi l}{\lambda} \frac{n^{3} B_{6}}{2}=+\frac{2 \pi l}{\lambda} \frac{n^{3}}{2}\left(r_{41} E\right)$

where $l$ is the thickness of the sample $\lambda$ the wave-length of the laser. 
If the sample is multidomain $r_{41}$ must be replaced by its mean value

$$
\left\langle r_{41}\right\rangle=\frac{1}{l} \int r_{41}(u) \mathrm{d} u \quad(u \text { along the beam }) .
$$

Unfortunately the formula (3) is valid only in the case of a perfect cubic crystal. As previously shown [13] $\mathrm{NH}_{4} \mathrm{Cl}$ single crystals have a very low elastic limit and slip bands parallel to $\{100\}$ type planes are systematically observed (even at room temperature); they induce compressive and tensile stresses $\left(\sigma_{1}, \sigma_{2}\right.$ and $\sigma_{3}$ relative to the cubic axes). Furthermore the heterophase structure which appears at the first order phase transition, involves plastic deformations inside $\{111\}$ plate like nuclei. A memory of these deformations is observed to persist for a long time below and above the temperature transition. These transition induced deformations are even more troublesome than the initial ones because they involve shear stresses, so that now a principal optical direction is found near $\langle 111\rangle$ directions, which is different from that of the electro-optic effect which is $\langle 001\rangle$ as shown in figure $1 a$ and $1 b$. Thus a crystal with internal stresses presents a complicated inhomogeneous birefringence pattern. Furthermore the birefringence even changes along the light beam. For a thin beam the sample can be decomposed into a succession of small homogeneous platelets, the total effect of which is equivalent to the effect of a homogeneous birefringent plate producing a retardation $\phi$ and with a principal direction making an angle $\alpha$ with the $\langle 001\rangle$ axis.
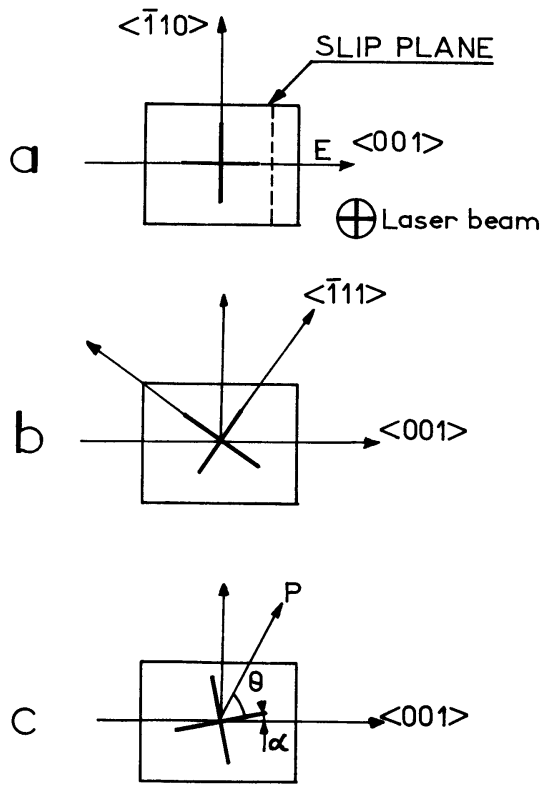

FIG. 1. - Orientation of the principal optical directions in a crystal of $\mathrm{NH}_{4} \mathrm{Cl}$ for a light beam propagating along $\langle 110\rangle$ with stress birefringence produced by : $a$ ) Preexisting $\{001\}$ slip bands or produced by an electric field $E$ along $\langle 001\rangle ; b$ ) Plastic deformation along $\{\overline{1} 11\}$ after the transition; $c$ ) The simultaneous presence of these two effects. (P is the polarizer direction.)
The light beam is linearly polarized by a polarizer $\mathrm{P}$, making an angle $\theta$ with the principal directions of the crystal (Fig. 1c). When an electric field $E$ is applied along $\langle 001\rangle$ both $\phi$ and $\theta$ change. If this field $E_{\omega}$ (in general modulated at frequency $\omega$ ) is small we can write :

$$
\begin{aligned}
& \phi=\phi_{0}+\left(\frac{\partial \phi}{\partial E}\right) E_{\omega}+\cdots=\phi_{0}+\phi_{\omega}+\cdots \\
& \theta=\theta_{0}+\left(\frac{\partial \theta}{\partial E}\right) E_{\omega}+\cdots=\theta_{0}+\theta_{\omega}+\cdots
\end{aligned}
$$

$\phi_{\omega}$ and $\theta_{\omega}$ are proportional to $r_{41}$ since the electrooptic effect is always produced by the term $r_{41} E$, but the value of these proportionality coefficients depends on $\phi_{0}$ and $\theta_{0}$ which change with temperature and field. Preliminary experiments, and calculations [23] have shown that the effect of rotation of the principal axis is quite important and must be eliminated. In order to do this we have used the following method. A polarizer, the sample, a $\lambda / 4$ plate and an analyser are disposed along the beam of a laser. The polarizer and the analyser are crossed. One can easily show that the emergent intensity modulated at the frequency $\omega$ is [23] :

$I_{1}^{\omega}=\frac{I_{0}}{2}\left(\phi_{\omega} \sin 2 \theta_{0} \cos \phi_{0}+2 \theta_{\omega} \cos 2 \theta_{0} \sin \phi_{0}\right)$

if the $\lambda / 4$ slow axis is at $45^{\circ}$ from the polarizer axis and

$I_{2}^{\omega}=\frac{I_{0}}{2}\left(\phi_{\omega} \sin ^{2} 2 \theta_{0} \sin \phi_{0}+2 \theta_{\omega} \sin 4 \theta_{0}\left(1-\cos \phi_{0}\right)\right)$

if the $\lambda / 4$ axis is parallel to the polarizer axis.

If we change the setting of the polarizer and of the $\lambda / 4$ so that $\theta_{0}$ always equals $45^{\circ}$ the preceeding intensities become

$$
\begin{aligned}
& I_{1}^{\omega}=\frac{I_{0}}{2} \phi_{\omega} \cos \phi_{0}, \quad I_{2}^{\omega}=\frac{I_{0}}{2} \phi_{\omega} \sin \phi_{0} \\
& I^{\omega}=\left(\left(I_{1}^{\omega}\right)^{2}+\left(I_{2}^{\omega}\right)^{2}\right)^{1 / 2}=\frac{I_{0}}{2} \phi_{\omega} \sim r_{41}(T) .
\end{aligned}
$$

With this method the variation of $\phi_{0}$ does not matter, and it is necessary only to follow the orientation of the principal axis of the sample.

3. Experimental set-up and results. - 3.1 EXPERIMENTAL SET-UP. - $\mathrm{NH}_{4} \mathrm{Cl}$ single crystals were grown in our laboratory by slow cooling of aqueous solutions with urea as habit modifier [24]. $4 \times 4 \times 4 \mathrm{~mm}^{3}$ samples were cut with a diamond saw (very slowly to reduce induced stresses), with $\{110\},\{1 \overline{10}\}$ and $\{001\}$ polished faces. Gold electrodes were evaporated on $\{001\}$ faces and were used to apply a static electric field (up to $15 \mathrm{kV} / \mathrm{cm}$ ) and an a.c. electric field up to $1 \mathrm{kV} / \mathrm{cm}$ peak to peak at $1 \mathrm{kHz}$. 
A cryostat with 4 optical windows, cooled by an electric refrigerator, was specially built to allow application of uniaxial stress up to $200 \mathrm{~N} / \mathrm{cm}^{2}$ and of high voltage electric fields. The sample is generally immersed in a small glass cell filled with silicon oil to improve the optical surface quality and to prevent electric discharge at high voltage. This cell is enclosed in a ceramic cylinder situated inside a large copper block. The temperature can be regulated with a $0.005^{\circ} \mathrm{C}$ stability and it can be linearly swept (from $0,1^{\circ} \mathrm{C} / \mathrm{h}$ to $2^{\circ} \mathrm{C} / \mathrm{h}$ ).

Special care was taken to avoid parasitic birefringence from the windows : the entrance window was replaced by a rotating sheet polarizer and the exit window was a thin $(0.1 \mathrm{~mm})$ microscopic glass slide. For some experiments the glass cell was removed to reduce parasitic birefringence. The birefringence pattern and the domain structure under applied field could be observed with a microscope having a long focal objective and a movable mirror, with white light illumination of the sample placed between crossed polarizers.

As explained before, to make a good measurement of the electro-optic effect, we must first determine the orientation of the principal direction of the crystal, at the beam position.

This was obtained with the following set up (Fig. 2a) : a $2 \mathrm{~mW} \mathrm{HeNe}$ laser as light source, a polarizer $P$, the sample $C$, a photoelastic modulator $M$ producing a birefringence modulation $\phi_{M}$ at a frequence $\omega_{M}$ an analysor $A$ parallel to $\langle 1 \overline{1} 0\rangle$ and a photodiode $\mathrm{Ph}$. The modulated intensity is [23] :

$$
I_{\omega_{M}}=\frac{I_{0}}{2} \phi_{M} \sin 2 \theta_{M} \sin \phi \sin 2 \theta
$$
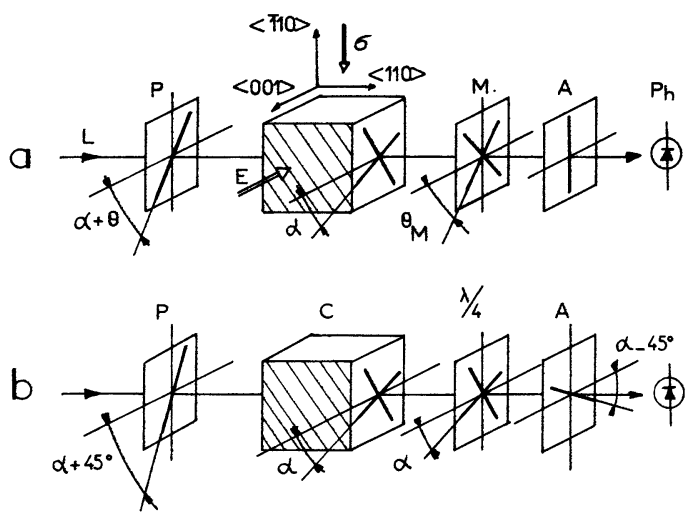

FIG. 2. - Optical apparatus for birefringence measurement : a) Determination of the principal optic axis orientation ; $b$ ) Measurement of the electro-optic effect; $\mathrm{L}: \mathrm{HeNe}$ laser, $\mathrm{P}:$ polarizer, $\mathrm{C}: \mathrm{NH}_{4} \mathrm{Cl}$ crystal, $\mathrm{M}$ : modulator, $\mathrm{A}:$ analyser, $\mathrm{Ph}:$ photodiode, $\mathrm{E}$ : electric field, $\sigma:$ stress.

where $\theta$ is the angle between the polarizer and the slow axis of the crystal, and $\phi$ the retardation angle produced by the crystal. By rotating the polarizer, we can make this signal vanish when $\theta=0$, and so determine the position $\alpha$ of the crystal axis to better than $0.1^{\circ}$. In general the parasitic birefringence is very inhomogeneous, $\alpha$ varying within $\pm 15^{\circ}$ around a mean position for small translations of $0.1 \mathrm{~mm}$ of the laser beam which is focused to a diameter of $50 \mu$. To make the measurements, we searched for regions with a more uniform birefringence pattern and with optical axes near those produced by the electric field. Then we checked that the orientation $\alpha$ of the neutral lines changes by less than $1^{\circ}$ as long as the crystal remains in one phase, while at the transition there is a change of several degrees between the two phases. The $\phi_{0}$ measured by the de Senarmont method [25] shows similar variations.

When the principal axis orientation had been determined, we placed the crossed polarizer and analyser at $45^{\circ}$ from these axes, and replaced the modulator by a $\lambda / 4$ plate with axis parallel to the crystal axis (Fig. 2b). Then we measured the electrooptic effect using a modulating electric field applied to the crystal by the method given in part 2 .

In making the measurement in a position where the effect of the electric field on the principal axis orientation is small, we checked experimentally that when $\theta_{0} \sim 45^{\circ}$ and $\phi_{0}<20^{\circ}$, equation (5) can be reduced to its first term directly giving $r_{41}$ with the same result as with equation (8).

\subsection{EXPERIMENTAL RESULTS OF ELECTRO-OPTIC} MEASUREMENTS. - Among our measurements we can separate 3 cases depending upon the domain structure.

a) The sample has many small domains whose electro-optic effects are partially cancelled so that the mean electro-optic effect is small. There is a weak signal $I_{\omega}$ which varies rapidly from point to point and the domains are too small to be directly seen.

b) The crystal fortuitously has one family of big domains with domain walls parallel to $\{111\}$ or $\{1 \overline{1} 1\}$. If the domains are broad enough and cross the whole sample parallel to the $\langle 110\rangle$ beam direction we have the maximum possible electro-optic effect. Then the electro-optic effect with a static field of $15 \mathrm{kV} / \mathrm{cm}$ is larger than the parasitic birefringence and tends to impose the principal direction. In this case the domains are directly visible [13], and the electro-optic effect can be measured either directly with the static field, or with a modulated field added to the static one. The corresponding signal is about 50 times that of case a. The phase of $I_{\omega}$ changes by $180^{\circ}$ when the laser beam is swept across a domain wall, the amplitude remaining nearly constant. Nevertheless one cannot be sure that only one type of domain wall orientation is present, because we can see only 2 of the $4\langle 111\rangle$ wall orientations.

c) The crystal is made single domain by the simultaneous application of a d.c. electric field

$$
E_{z}=15 \mathrm{kV} / \mathrm{cm}
$$

and of an uniaxial stress $\sigma_{x y}=150 \mathrm{~N} / \mathrm{cm}^{2}$. Then as in 
case $b$ the electro-optic effect is strong. The variation of the electro-optic effect in these 3 cases as a function of temperature are in good agreement as is shown in figure 3 . In the coexistence region around the transi-

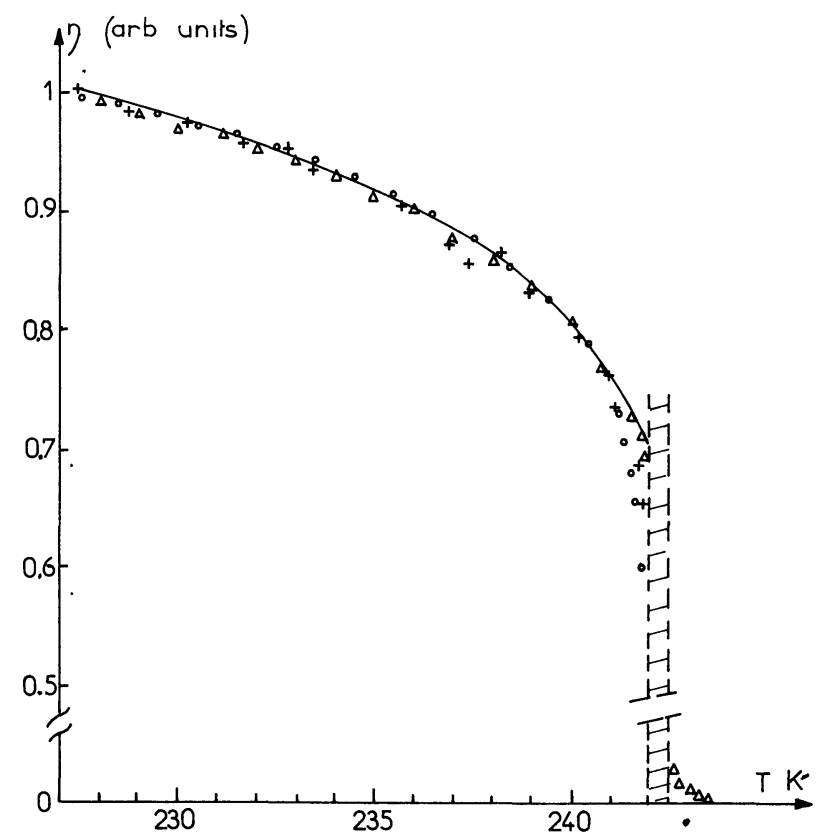

FIG. 3. - Temperature variation of the electro-optic effect for 3 different experimental cases : (measurements cannot be made inside the dashed zone, which corresponds to the coexistence region) : $O$ Inside a big domain; $\Delta$ In a crystal with many small domains ; + In a single domain crystal obtained by the application of an electric field along $\langle 001\rangle$ and of a stress $\sigma$ along $\langle\overline{1} 10\rangle$.

The full curve corresponds to the fit with exponent 0.125 .

tion, the electro-optic effect variation is continuous but changes greatly from sample to sample. In this region there is a very intense small angle light scattering $I_{\mathrm{s}}$ (Fig. 4). (Experiments of light beating spectroscopy have shown that this light scattering is essentially static [26].) The transmitted intensity is reduced by an order of magnitude, so that optical measurements in this region are too greatly perturbed to be of any value. Similar, but less intense light scattering has been observed near the $\alpha-\beta$ phase boundary in quartz [27]. Outside the coexistence region all the measured curves have the same temperature variations, which indicates that the domain structure is stable and influences only the magnitude of the average electro-optic effect, and not its temperature variation.

The curves drawn are the same with or without an applied stress and an electric field showing that linear coupling between the order parameter $\eta$ and $\sigma_{x y} E_{z}$ is undetectable in our experiment. (We have only observed the small elevation of transition temperature produced by application of the stress.) The small value of the coupling between $\eta$ and $\sigma_{x y} E_{z}$ is confirmed by difficulties encountered in changing the domain structure. When $\sigma_{x y} E_{z}$ was applied in the low temperature phase after transition, we observed no change. We obtained a single domain crystal only

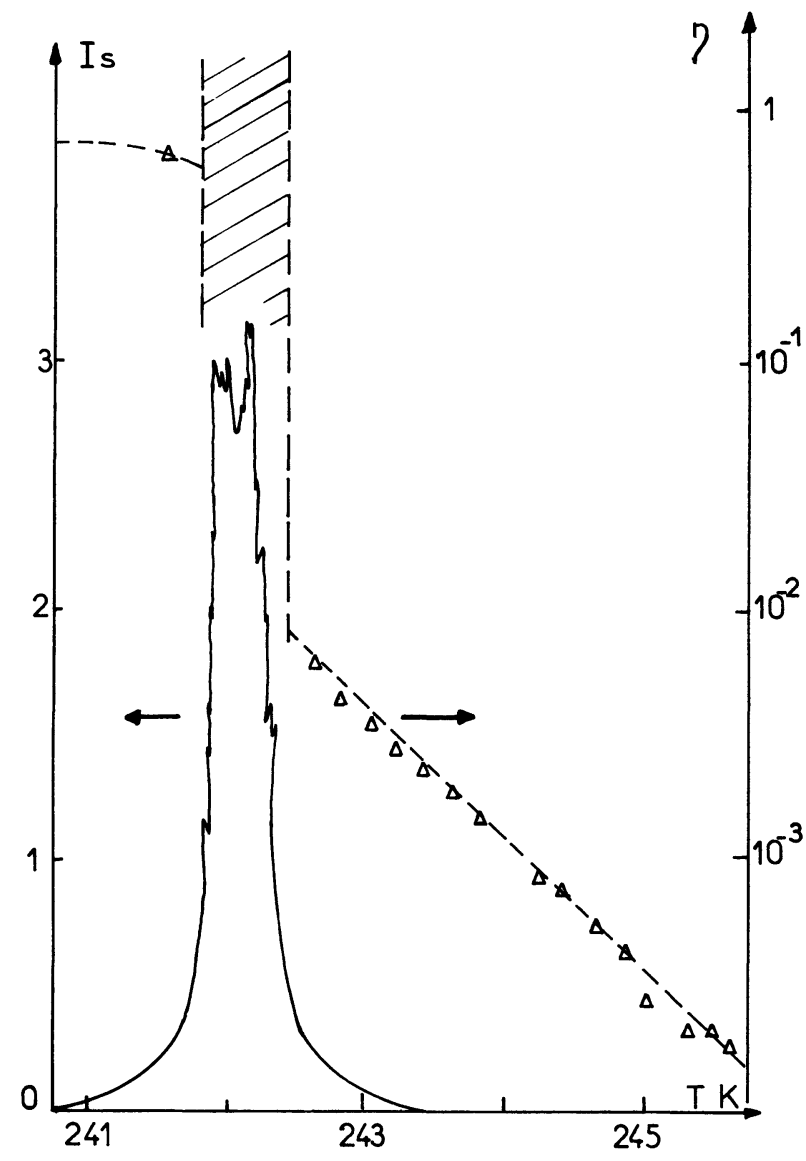

Fig. 4. - Temperature variations of the intensity of small angle light scattering $I_{\mathrm{s}}$ (full curve) and the electro-optic effect (dashed curve) in the vicinity of the transition temperature in a polydomain sample (the electro-optic effect is plotted with a logarithmic scale to show its exponential temperature dependence). The units are arbitrary.

when $\sigma_{x y} E_{z}$ was applied during the first transition of a virgin crystal. Then, in the low temperature phase, the crystal keeps the same single domain state, even if the field is reversed. Changes in this single domain crystal were obtained only with a new transition under the opposite field, and then the crystal was only partially switched.

The absolute value of $r_{41}$ has been measured in case $b$ ) and $c$ ) with a static electric field of $15 \mathrm{kV} / \mathrm{cm}$ by the de Senarmont method at $229 \mathrm{~K}$. We obtain :

$$
\begin{aligned}
r_{41} & \left.\sim 1.5 \times 10^{-10} \mathrm{~cm} / \mathrm{V} \text { in case } b\right) \\
r_{41} & \left.\sim 2.0 \times 10^{-10} \mathrm{~cm} / \mathrm{V} \text { in case } c\right) .
\end{aligned}
$$

The difference between the two measurements can be produced by domains which cannot be seen in our experiment. Except for a recent measurement on a single domain crystal by Konak $[18,19]$ who found $r_{41}=3 \times 10^{-10} \mathrm{~cm} / \mathrm{V}$, the only previous reported measurement is $r_{41}=1.46 \times 10^{-10} \mathrm{~cm} / \mathrm{V}$ at $233 \mathrm{~K}$ without mention of the domain problem [28]. So there is only agreement for the order of magnitude of $r_{41}$ not for the exact value. 
In all the experiments a tail of $r_{41}$ above the transition is present, as in previous piezoelectric [11] and second harmonic measurements $[16,17]$. This residual electro-optic effect has a great spatial variation, but for a fixed position it decreases exponentially as a function of the temperature (Fig. 4). When a static electric field is applied we have observed a temperature independent quadratic electro-optic effect in the high temperature phase which produces a modulated intensity :

$$
I_{\omega}=\frac{I_{0}}{2} \frac{2 \pi l}{\lambda} n^{3}\left(R_{11}-R_{12}\right) E_{0} E_{\omega} \cos \phi_{0}
$$

where $R_{11}$ and $R_{12}$ are coefficients for the quadratic effect. As we have found that this quadratic effect with $E_{0}=15 \mathrm{kV} / \mathrm{cm}$ is about $10^{-3}$ of the linear effect we have at $229 \mathrm{~K}$

$$
R_{11}-R_{12} \sim 10^{-17}(\mathrm{~cm} / \mathrm{V})^{2} .
$$

In addition to these electro-optic measurements, we have made some measurements with second harmonic generation in polydomain crystals (case $a$ ). The non linear susceptibility $\chi_{14}$ is in a first approximation proportional to the order parameter. The second harmonic intensity $I_{2 \omega} \sim \chi_{14}^{2}$ has a smooth temperature dependence as it is the sum of the intensities produced by a great number of small domains [17]. Our second harmonic measurements are in good agreement with those of Steinbrener et al. [17] and with our electro-optic measurements (Fig. 5). We have also observed [23] the non linear diffraction by domains [29] and the exponential tail a few degrees above the transition temperature $[16,17]$.

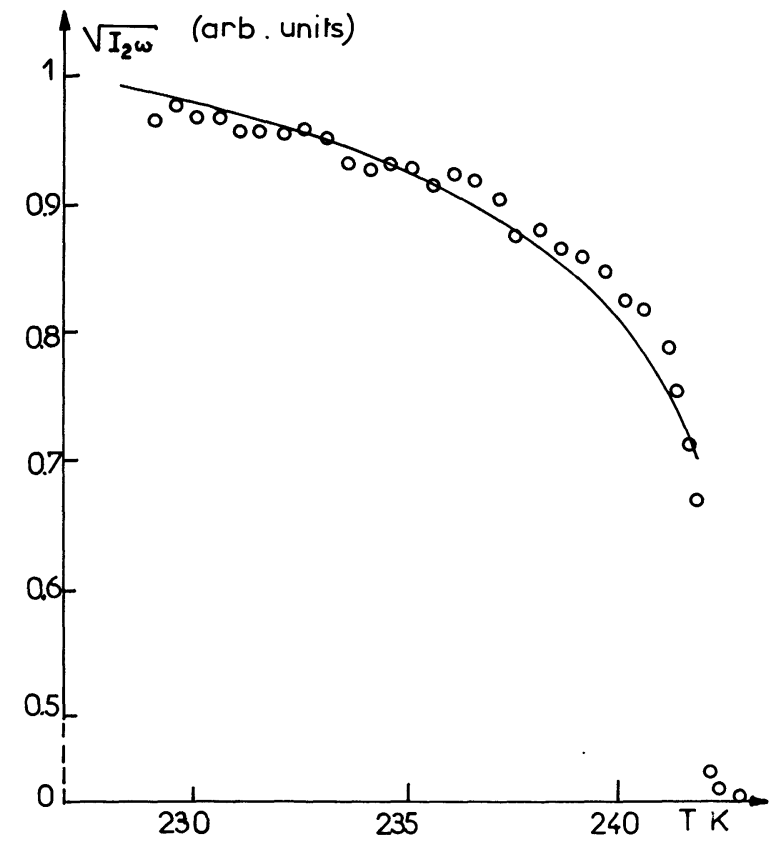

FIG. 5. - Temperature dependence of the square root of the second harmonic $I_{2 \omega}$ intensity (dots). The full curve corresponds to the fit to our electro-optic measurements with exponent $\beta=0.125$ (same curve as in Fig. 3).
4. Discussion. - A comparison of the various electro-optic, second harmonic generation and piezoelectric data is given in figure 6. With the exception of the piezoelectric measurements of Molher and Pitka [15], all these results are in good agreement, showing that all are proportional to the order parameter, in the temperature range considered.

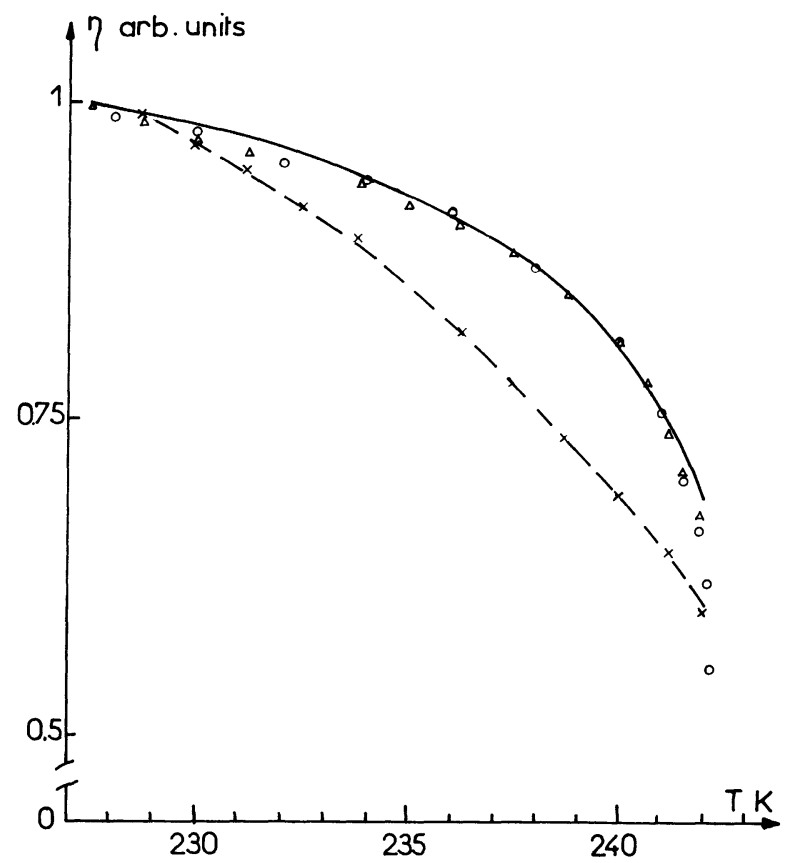

FIG. 6. - Comparison of various measurements of the order parameter $\eta$ in $\mathrm{NH}_{4} \mathrm{Cl}: \times$ Piezoelectric effect in a monodomain sample (Molher and Pitka [15]); $\Delta$ Piezoelectric effect in a polydomain sample (Bruins and Garland [11]); O Second harmonic measurement (Steinbrener and Jahn [17]). The full curve corresponds to the fit to our electro-optic measurement with exponent $\beta=0.125$ (same curve as in Fig. 3 and 5).

To interpret the temperature variation of the order parameter in a first order transition two kinds of description have been used : the Landau theory and critical exponent laws.

In the Landau theory [30] the free energy $G$ is developed in increasing powers of $\eta$ (only even powers are present in $\mathrm{NH}_{4} \mathrm{Cl}$ due to symmetry consideration) :

$$
\begin{aligned}
G=G_{0}+\frac{a}{2}( & \left.-T_{0}\right) \eta^{2}+\frac{b}{4} \eta^{4}+ \\
& +\frac{c}{6} \eta^{6}+\frac{d}{8} \eta^{8}+\frac{e}{10} \eta^{10}+\cdots
\end{aligned}
$$

The equilibrium value of $\eta$ is given by $\partial G / \partial \eta=0$.

If $b<0$ there is a first order transition.

The other description which has been proposed [31] is a critical exponent law :

$$
\eta=\eta^{*}+K\left(T^{*}-T\right)^{\beta}
$$

where $T^{*}$ is the transition temperature, and $\eta^{*}$ the value of $\eta$ for this temperature. This 4-parameter 
formula has been shown to have so much freedom for a fitting procedure that its physical signification is dubious [32]. So here we discard the $\eta^{*}$ term and use $\eta=K\left(T^{*}-T\right)^{\beta}$ as for a second order transition this procedure has some theoretical justification only near a multicritical point but this can be the case for $\mathrm{NH}_{4} \mathrm{Cl}$ since the transition becomes 2 nd order for relatively low pressure (1.5 kbars [8]) and still more for $\mathrm{ND}_{4} \mathrm{Cl}$ where the critical pressure is around 150 bars [1]. The exponents previously found for $\mathrm{ND}_{4} \mathrm{Cl}$ and $\mathrm{NH}_{4} \mathrm{Cl}$, for various experiments, are given in table $\mathrm{I}$.

\section{TABLE I}

Value of the critical exponent $\beta$ and of the critical temperature $T^{*}$ for the temperature variation of the order parameter $\left(\eta \sim\left(T^{*}-T\right)^{\beta}\right)$ found in different experiments (the temperature range used for the numerical fit is given in the last columns).

\begin{tabular}{|c|c|c|c|c|}
\hline & $\beta$ & $T^{*}$ & Reference & $T$ range $(\mathrm{K})$ \\
\hline & - & - & 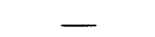 & \\
\hline $\mathrm{ND}_{4} \mathrm{Cl}$ & 0.153 & 248.86 & [1] & 225 to $T^{*}-1$ \\
\hline & 0.126 & 249.69 & [33] & 230 to 249.63 \\
\hline $\mathrm{NH}_{4} \mathrm{Cl}$ & 0.134 & 242.63 & [11] & 230.6242 .58 \\
\hline 4 & 0.136 & 242.617 & [17] & $232.4 \quad 242.26$ \\
\hline - & 0.33 & 244.78 & [15] & 242.3 \\
\hline
\end{tabular}

In addition to this power law Bruins and Garland [11] have also tried a fit according to Landau development of equation (11) up to $\eta^{10}$, and taking $c=d=0$ which would correspond to transition near a pentacritical point. This Landau fit is good for $\mathrm{ND}_{4} \mathrm{Cl}$ [33], where they found $\beta \sim 0.125$ but not for $\mathrm{NH}_{4} \mathrm{Cl}[11]$ where they found $\beta \sim 0.134$.

Steinbrener and Jahn [17] who found $\beta=0.136$ from their second harmonic measurements on $\mathrm{NH}_{4} \mathrm{Cl}$, followed a suggestion of Benguigui [10] and tried a fit to a Landau expansion up to $\eta^{8}$, with $b=0$. They then found a good fit with a small negative value of $c$, corresponding to a tetracritical point [17]. But in a recent paper Steinbrener showed that a good fit is also possible with a pentacritical point [34].

Following these trends we have analysed our data with critical exponent laws and with different kinds of Landau expansions. We have taken 55 points with a regular $0.25 \mathrm{~K}$ interval from 227.45 to $240.95 \mathrm{~K}$ in one of our measurements of the electro-optic effect, as a function of temperature. (Some of these points at $1 \mathrm{~K}$ intervals are given in table II.) At higher temperature, near the transition the measurements are perturbed by the coexistence state. Nevertheless, we have taken 6 more points between 241.20 and $241.85 \mathrm{~K}$ which were corrected for the laser beam attenuation. This correction increases here from 1.5 to $14 \%$. We think that at higher temperature the perturbation due to the coexistence state is too great to be accurately corrected.
TABLE II

Temperature variation of the order parameter (normalized to 1 at $227.45 \mathrm{~K})$ measured with the electrooptic effect. The last six points have been corrected (see text). The uncorrected data are given in parenthesis.

\begin{tabular}{rc}
$T$ & \multicolumn{1}{c}{$\frac{\eta}{1}$} \\
227.45 & 1 \\
228.45 & 0.993 \\
229.45 & 0.985 \\
230.45 & 0.976 \\
231.45 & 0.967 \\
232.45 & 0.957 \\
233.45 & 0.944 \\
234.45 & 0.931 \\
235.45 & 0.915 \\
236.45 & 0.899 \\
237.45 & 0.879 \\
238.45 & 0.853 \\
239.45 & 0.822 \\
240.45 & 0.781 \\
241.20 & $0.732(0.722)$ \\
241.45 & $0.721(0.689)$ \\
.55 & $0.718(0.670)$ \\
.65 & $0.710(0.647)$ \\
.75 & $0.700(0.627)$ \\
.85 & $0.695(0.608)$
\end{tabular}

(The intense light scattering being produced between 241.85 and $242.35 \mathrm{~K}$.)

We have first tried fits to a power law

$$
\eta=K\left(T^{*}-T\right)^{\beta}
$$

with the usual least square method minimizing

$$
\sigma^{2}=\frac{1}{n}\left(\sum_{1}^{n}\left(\eta_{\text {exp }}-\eta_{\text {theor }}\right)^{2}\right) .
$$

The results of these fits in different temperature ranges with $\beta$ free or fixed are given in table III. (There is a $64 \%$ probability that the parameters have a value inside the incertitude range given.)

The values found for the parameters are quite dependent upon the experimental results obtained in the vicinity of the transition temperature. This dependence is troublesome as the value of these points is affected by the coexistence state. Imposing $\beta=0.125=1 / 8$ increases the mean square deviation $\sigma$ very little. Imposing $\beta=1 / 6$ or $1 / 10$ increases $\sigma$ very much. Even bigger deviations would be produced with $\beta=1 / 3$ which is clearly excluded for our results. The curve corresponding to the fit with $\beta=0.125$ has been plotted in figures 3,5 and 6 as a reference curve. To show more clearly the deviations with the experimental results, we have plotted in figure 7 , as a function of temperature, the differences between all the experimental points used in the fitting procedure, 
TABLE III

Parameter values of fits, of our electro-optic data for the order parameter to a power law $\eta=K\left(T^{*}-T\right)^{\beta}$. ( $\sigma$ is the mean square deviation of $\eta$.) In the first three lines $\beta$ was free, while it was fixed at the indicated values in the last three lines. (There is a $67 \%$ probability that the parameters have a value inside the uncertainty range given.)

$\begin{array}{ccccccc} & \beta & T^{*}(\mathrm{~K}) & K & K^{-1 / \beta} & \sigma & T \text { range (K) } \\ 1 & 0.128 \pm 0.0017 & 242.62 \pm 0.04 & 0.710 \pm 0.003 & & 0.0034 & 227.4 \text { to } 241.85 \\ 2 & 0.125 \pm 0.0016 & 242.51 \pm 0.04 & 0.716 \pm 0.003 & & 0.0030 & 227.4 \text { to } 241.65 \\ 3 & 0.121 \pm 0.0013 & 242.32 \pm 0.04 & 0.725 \pm 0.003 & & 0.0023 & 227.4 \text { to } 241.45 \\ 4 & 1 / 6 & 243.69 \pm 0.07 & 0.638 \pm 0.001 & 14.77 & 0.0088 & 227.4 \text { to } 241.85 \\ 5 & 1 / 8 & 242.56 \pm 0.02 & 0.715 \pm 0.0005 & 14.62 & 0.0034 & 227.4 \text { to } 241.85 \\ 6 & 1 / 10 & 242.14 \pm 0.03 & 0.758 \pm 0.001 & 15.97 & 0.0100 & 227.4 \text { to } 241.85\end{array}$

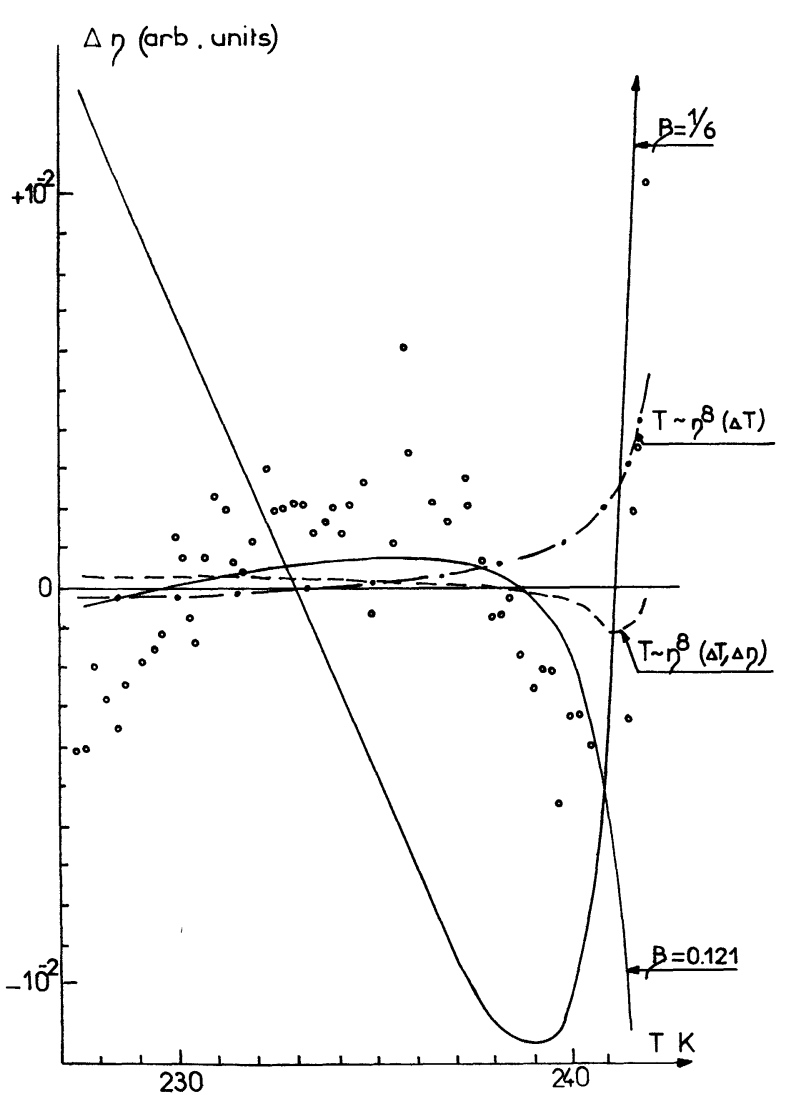

Fig. 7. - The differences $\Delta \eta$ between the experimental points (dots) and various curves are plotted as a function of temperature taking as reference line for $\Delta \eta=0$ the curve with exponent $\beta=0.125$ (same curve as in Fig. 3,5 and 6). Full curve : power law with $\beta=1 / 6$ and $\beta=0.121 ;-. .-. T \sim \eta^{8}(\Delta T)$ Landau fit, according to equation (13) keeping only the $\eta^{8}$ term, obtained in minimizing differences in $T$ only; - - $T \sim \eta^{8}(\bar{\Delta}, \Delta \eta)$ Landau fit, according to equation (13) keeping only the $\eta^{8}$ term, obtained in minimizing differences in $T$ and $\eta$.

and the curve with $\beta=0.125$. The differences with the $\beta=0.121$ and $\beta=1 / 6$ curves, corresponding respectively to line 3 and 4 of table III are also plotted. The small systematic deviation which appears between the curve $\beta=0.125$ and the experimental data, is reduced with the curve $\beta=0.121$ obtained in neglecting the 4 last points. So it seems that the correction for these points has been a little too great. However as these differences are of the order of $10^{-2}$ which is of the order of the data accuracy we have left our data as they are. We have also made some attempts with fits to the Landau theory. Minimizing $G$ given in the equation (11) leads (with $a=1$ ) to the relation

$$
T=T_{0}-\left(b \eta^{2}+c \eta^{4}+d \eta^{6}+e \eta^{8}+\cdots\right) .
$$

In this form $T$ appears as a polynom in $\eta$ which can be easily fitted with programs already developed in our laboratory by Dr. Lombardi. When one keeps only one power $\eta^{2 n}$ in equation (13), one obtains the reciprocal function of the previous power law with $\beta=1 / 2 n$. In this case we can compare the results of the fits given in table IV with those of table III. The values of the parameters are a little different. Some deviation also appears between these curves as shown by the curve $T \sim \eta^{8}(\Delta T)$ of figure 7 . To reduce the difference between the two procedures, we used for the fits with the $T(\eta)$ polynom, a programme written by Dr. Lombardi, which can take into account incertitudes $\Delta T$ on $T$ and $\Delta \eta$ on $\eta$ and which minimizes a distance $\sigma^{\prime}$ between the experimental points and the curve given by :

$\sigma^{\prime 2}=\frac{1}{n} \sum_{1}^{n}\left[\left(\frac{\eta_{\exp }-\eta_{\text {theor }}}{\Delta \eta}\right)^{2}+\left(\frac{T_{\text {exp }}-T_{\text {theor }}}{\Delta T}\right)^{2}\right]$

as explained in Wolberg's book [35].

The following results, given in table $\mathrm{V}$, have been obtained with this programme. We first investigated the possibility of an ordinary first order transition, which does not give good agreement. Going to a $T(\eta)$ polynoms with a $\eta^{6}$ term, we found that a better agreement is obtained with the $\eta^{4}$ terms as used by Steinbrener et al. (line 2) than with a $\eta^{2}$ term. A very good agreement is obtained with all the terms with power $2,4,6$ but now there is one more free parameter and also the value of $T_{0}$ is very far from the last experimental point. Now going up to polynoms in $\eta^{8}$ as tried by Garland et al. $[33,11]$ we found the best fit for a 3 term polynom with power 2 , and 8 in $\eta$ (line 5). The coefficient $b$ of $\eta^{2}$ is quite small with a 


\section{TABLE IV}

Parameter values of the fits of our electro-optic data for the order parameter to a polynom $T=T_{0}-\left(d \eta^{6}+e \eta^{8}+f \eta^{10} \ldots\right)$ minimizing $\sigma_{T}$ which is the mean square deviation of $T$. (The parameters $d$, e, $f$ correspond to $K^{-1 / \beta}$ of table 3 for $\beta=1 / 6,1 / 8,1 / 10$.)

$\begin{array}{cccccc}T_{0}(\mathrm{~K}) & d & e & f & \sigma_{T} & T \text { range } \\ - & - & - & - & - & - \\ 244.29 \pm 0.14 & 15.8 \pm 0.2 & 0 & 0 & 0.45 & 227.4 \text { to } 241.85 \\ 242.60 \pm 0.04 & 0 & 14.69 \pm 0.07 & 0 & 0.17 & 227.4 \text { to } 241.85 \\ 241.55 \pm 0.06 & 0 & 0 & 14.1 \pm 0.1 & 0.24 & 227.4 \text { to } 241.85\end{array}$

\section{TABLE V}

Parameter values of the fits of our electro-optic data for the order parameter to a polynom $T=T_{0}-\left(a \eta^{2}+\cdots+e \eta^{8}\right)$ minimizing $a$ "distance " $\sigma$ ' between the experimental points and the theoretical curve (see text).

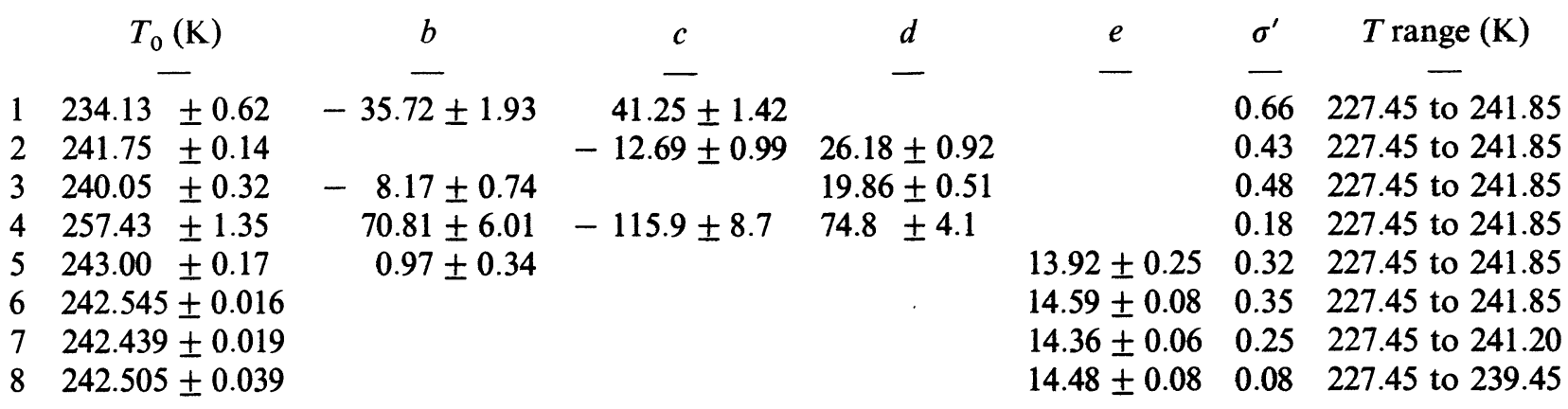

large relative uncertainty (around $33 \%$ ), which suggests that a polynom with $b=0$ would also be a good fit. This fit with only two parameters is represented by the curve $T \sim \eta^{\gamma}(\Delta T, \Delta \eta)$ in Fig. 7 and is nearly as good as the previous one. The parameter values are also very stable when the temperature range used for the fit is reduced (lines 6 to 8 ). This case corresponds to a pentacritical point and to the exponent $\beta=0.125$.

Recently Poppe and Huller [36] have suggested that near a tricritical point the coefficient $b$, which becomes zero at the tricritical point, is not constant, and that it would be necessary to introduce a temperature variation of $b$. This procedure increases the number of unknown parameters and we have made no attempt to test it with the present data; however we think that we shall be able to check this hypothesis with new data on the pressure dependence of the order parameter which are presently being measured by second harmonic generation.

Although our experimental results are very similar to the previous ones as shown in figure 6 , the numerical results are a little different, in particular for $\beta$ which is smaller here than in previous experiments where $\beta$ was around 0.135 .

For the experiment of Bruins and Garland [11] this greater value of $\beta$ is probably related to the fact that they took into account points very near the transition with $\Delta T$ as low as $5 \times 10^{-2} \mathrm{~K}$. These points are certainly inside the coexistence region, and are perturbed by this state. In $\mathrm{ND}_{4} \mathrm{Cl}$ [33] which is closer to the multicritical point, coexistence effects are expected to be small, as corroborated by the smaller tail. Indeed $\beta=0.125$ and a good fit to a pentacritical point is found. So with nearly the same experimental results, different fits can be obtained, which show that they are quite sensitive to small variations in the data, particularly for the points near the transition. Then the question arises : how seriously shall we consider the value $\beta=0.125$. It appears that good representations of our data are obtained with only two free parameters, for this exponent value. (It would have been interesting to have results at a lower temperature to see if this power law is always true.) However the interpretation of the transition as pentacritical seems difficult. There are only two experimental fields acting on the transition, temperature and pressure. One can conjecture that another field is associated with the anti-parallel order of the tetrahedra, which exist in $\mathrm{NH}_{4} \mathrm{Br}$. But this would only lead to a tetracritical point. Another field is needed to reach a pentacritical point.

If only data at atmospheric pressure were known, one might conclude that this is only a first-order transition, and that the exponent value $\beta=0.125$ is spurious (a curve with a small temperature variation corresponding to a low value of the exponent). However as it is known that under pressure the transition becomes continuous, this value of $\beta$ may be of some value. A complete answer can only be 
obtained with new measurements of the order parameter under pressure, so that critical exponents can be deduced without ambiguity. A comparison with the recent measurements of the order parameter in KDP near a tricritical point would be of particular interest $[37,38]$.

In a recent work [39] Garland et al. have measured the critical exponent $\alpha^{\prime}$ describing the divergence of the heat capacity of $\mathrm{NH}_{4} \mathrm{Cl}$ and $\mathrm{ND}_{4} \mathrm{Cl}$ under pressure. However they say that their results cannot resolve the question of the character of the multicritical point.

Another point of concern in the $\mathrm{NH}_{4} \mathrm{Cl}$ transition is the existence of residual order above the transition temperature, as shown by the tail observed in figure 4 . This feature was discovered by Freund in S. H. experiments [40] and he found that this residual order decreases exponentially when the temperature increases.

Such an exponential tail has been found systematically in later works as is shown in figure 8 where the results of different experiments have been plotted with a semi-logarithmic scale. Excepting the first cooling for which the tail is generally smaller and may have a different behaviour, these curves are fairly reproducible for successive transitions. Surprisingly all these results, obtained with different techniques on different samples, lead to a similar exponential temperature dependence. On heating and on cooling,

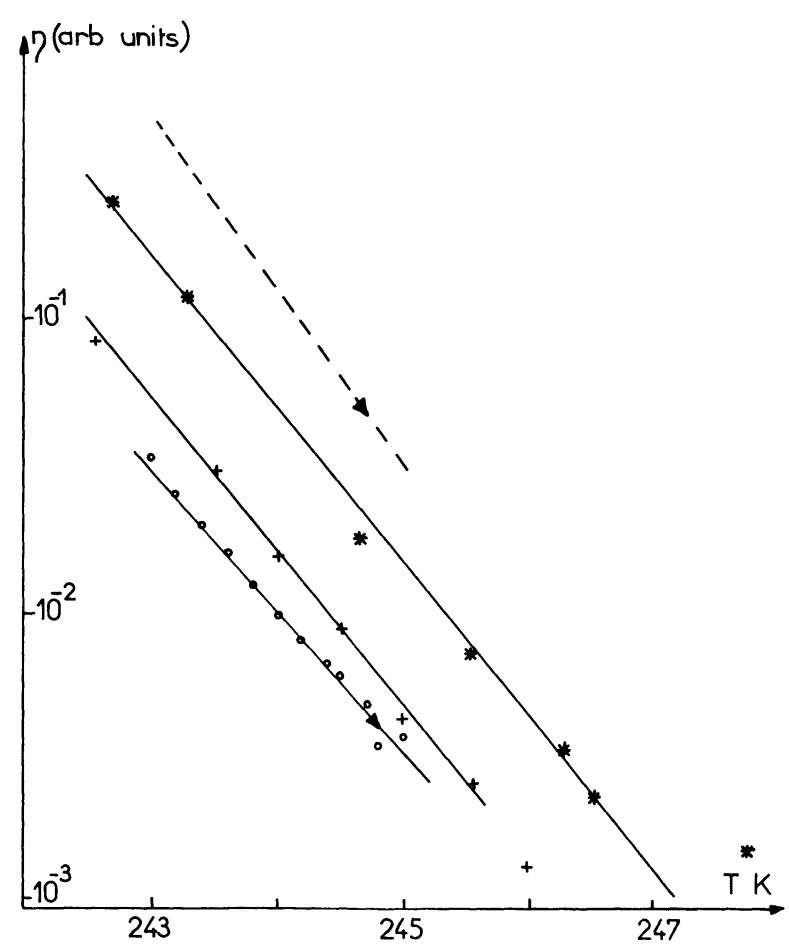

FIG. 8. - Exponential dependence of the residual long range order in the tail as a function of temperature above the mean transition temperature for different experiments : O Electro-optic effect (this work); + Second harmonic (square root of the intensity of diffracted second harmonic at its maximum), this work $; *$ Second harmonic (square root of the intensity of diffracted second harmonic at its maximum), ref. [16]; - - Piezoelectric effect (ref. [11]). (The relative positions of these curves are arbitrary along the $\eta$ axis.) the curves have the same slope, their main difference is a translation of about $1 \mathrm{~K}$ which corresponds to the thermal hysteresis.

In a recent hyper Raman experiment, some residual elastic second harmonic scattering was observed even at room temperature [41]. From his first experiment made on powder, Freund interpreted this phenomena as critical $\mathrm{S}$. $\mathrm{H}$. scattering produced by order parameter fluctuations [40]. So this S. H. scattering would have been an early manifestation of the central peak phenomena [42]. However in later work on $\mathrm{NH}_{4} \mathrm{Cl}$ single crystals [16], Freund et al. found that the scattering was essentially produced at small angle, showing that it was produced by long range order over several tens of microns which cannot be interpreted as a microscopic effect. So, as in the central peak history, the influence of defects must be considered [42], and in particular for this case, the effect of internal stresses. We have shown already that such stresses are systematically found in $\mathrm{NH}_{4} \mathrm{Cl}$, either preexistent in the crystal and depending on its history, or produced by plastic deformation during the transition itself [13]. The later explanation is probably better, in order to explain the reproducible results between different samples. In general the stresses are of the same order of magnitude $\left(30 \times 10^{6}\right.$ dynes $\left./ \mathrm{cm}^{2}\right)$ but near the dislocation, the stresses can be far greater $\left(5 \times 10^{8}\right.$ dynes $/ \mathrm{cm}^{2}$ at $250 \AA$ from the dislocation core) which can produce a local increase of the transition temperature of about $2 \mathrm{~K}$ and thus, have a great effect for the nucleation of the new phase. Such an influence of dislocations has already been conjectured by Bartis [43]. But as the stress field around a dislocation decreases as $1 / r$, he found that the S. H. intensity changes as $\left(T-T_{\mathrm{c}}\right)^{-3}$ which is not in good agreement with the experimental exponential dependence. It seems that something more must be added to the internal stress hypothesis. Often in phase nucleation theory there is a potential barrier $U$ which is overcome by a thermal activation process given by $\mathrm{e}^{-\boldsymbol{U} / \boldsymbol{k} T}$ [44]. In our case it would be sufficient, in the small temperature range considered, for $U$ to have a nearly linear temperature dependence. In a first order phase transition the activation energy is associated either with the nucleation or with the growth of nuclei. Both models have been developped in connection with martensitic transformations. In the first case the potential barrier is produced by a surface energy term, but usually this leads to a barrier which is far too great to be overcome by thermal fluctuation. To avoid this problem it is necessary to introduce preexistant nuclei [45], or to help the nucleation by the favorable action of defects (such as dislocations [46] or stacking faults [47]).

In the second model the activation process may appear in the further growth of these nuclei. This can happen with the nucleation of dislocation loops at the interface between the two phases [48]. We have considered the application of such models to $\mathrm{NH}_{4} \mathrm{Cl}$. 
For the transition on cooling the orders of magnitude are reasonable, but we do not see how, on heating, the same variations can be obtained. This quasi reversibility on cooling and on heating seems incompatible with an activation process. It would be necessary to introduce a mechanism giving some kind of equilibrium between the transition energy and deformation energy (elastic and plastic) which would be more reversible, but it seems difficult to find an exponential dependence, unless we suppose that the transition temperatures already have an exponential distribution.

Further experiments are planned to give more information on this puzzling question.
5. Conclusion. - In this paper we have presented a new measurement of the order parameter of $\mathrm{NH}_{4} \mathrm{Cl}$ using the electro-optic effect.

An exponent $\beta \sim 0.125$ gives a good numerical fit to our data, but we think that this result is not sufficient to establish firmly that the transition is close to a pentacritical point: Before accepting this conclusion further experimental results are needed, and in particular measurements of the order parameter under high pressure.

Acknowledgments. - The authors wish to thank Dr. Lombardi for his assistance in the curve fitting and P. Palleau for this help in the experimental work.

\section{References}

[1] Yelon, W. B., Cox, D. E., Kortman, P. J. and Daniels, W. B., Phys. Rev. B 9 (1974) 4843.

[2] Garland, C. W. and Renard, R., J. Chem. Phys. 44 (1966) 1120.

[3] Huller, A., Z. Physik 270 (1974) 343.

[4] Terauchi, H., Noda, Y. and Yamada, Y., J. Phys. Soc. Jpn 32 (1972) 1560.

[5] Jahn, I. R., Brunskill, I. H., Bausch, R. and Dachs, H., J. Phys. C 8 (1975) 3280.

[6] Goto, T. and KamiYoshi, K. I., J. Phys. Soc. Jpn 42 (1977) 1924.

[7] Couzi, M., Denoyer, F. and Lambert, M., J. Physique 35 (1974) 753.

[8] Weiner, B. B. and Garland, C. W., J. Chem. Phys. 56 (1972) 155.

[9] Amitin, E. B., Kobalevskaya, Yu. A. and Paukov, I. E., Zh. Eksp. T. Fiz. 71 (1976) 700, Sov. Phys. JETP 44 (1976) 368.

[10] Benguigui, L., Solid State Commun. 20 (1976) 173.

[11] Bruins, D. E. and Garland, C. W., J. Chem. Phys. 63 (1975) 4139.

[12] Boccara, N., Symétrie brisée (Hermann, Paris), 1976.

[13] Pique, J. P., Dolino, G. and Vallade, M., J. Physique 38 (1977) 1527

[14] Aizu, K., J. Phys. Soc. Jpn 34 (1973) 121.

[15] Mohler, E. and Pitka, R., Solid State Commun. 14 (1974) 791.

[16] Freund, I. and Kopf, L., Phys. Rev. Lett. 24 (1970) 1017.

[17] Steinbrener, S. and Jahn, I. R., J. Phys. C 11 (1978) 1337.

[18] Cited by FouseK, J., Ferroelectrics (to be published).

[19] Konak, C., Budik, L. and Novak, K., Phys. Status Solidi (a) 45 (1978) K-63

[20] Pique, J. P., Dolino, G. and Vallade, M., Ferroelectrics (to be published).

[21] Nye, J. F., Physical properties of crystal (Oxford University Press, London), 1972.

[22] Narasimhamurty, T. S., Curr. Sci. 5 (1954) 149.

[23] Pique, J. P., Thesis, Grenoble (1978) (unpublished).
[24] Bunn, G. W., Proc. Roy. Soc. London $A 141$ (1933) 567.

[25] Jerrard, H. G., J. Opt. Soc. Am. 44 (1954) 634.

[26] Adam, M. and Searby, G. M., Phys. Status Solidi (a) 19 (1973) 185.

[27] Dolino, G. and Bachheimer, J. P., Phys. Status Solidi (a) 41 (1977) 673.

[28] Vasell, M. and Conwell, E. M., Phys. Rev. A 140 (1965) 2110.

[29] Freund, I., Phys. Rev. Lett. 21 (1968) 1404.

[30] LANDAU, L. and LIFCHITZ, E., Physique statistique (MIR, Moscou), 1967.

[31] Hochli, U. T. and Scott, J. F., Phys. Rev. Lett. 26 (1971) 1627.

[32] BachHeimer, J. P. and Dolino, G., Phys. Rev. B 11 (1975) 3195.

[33] Garland, C. W., Bruins, D. E. and Greytak, T. J., Phys. Rev. B 12 (1975) 2759.

[34] Steinbrener, S., Solid State Commun. (1978) (to be published)

[35] Wolberg, J. R., Prediction analysis (Van Nastrand), 1967.

[36] Poppe, U. and Huller, A., J. Phys. C 11 (1978) 245.

[37] Schmidt, V. H., Western, A. B. and Baker, A. G., Phys. Rev. Lett. 37 (1976) 839.

[38] Bastie, P., Vallade, M., Vettier, C. and Zeyen, C. M. E., Phys. Rev. Lett. 40 (1978) 337.

[39] Garland, C. W. and Baloga, J. D., Phys. Rev. B 16 (1977) 331.

[40] Freund, I., Phys. Rev. Lett. 19 (1967) 1288.

[41] Dines, T. J., French, M. J., Hall, R. J. B. and Long, D. A., Proceedings of the 5th conf. Raman. Spectroscopy (1976) p. 707.

[42] Halperin, B. I. and Varma, C. M., Phys. Rev. B 14 (1976) 4030.

[43] Bartis, F. J., Phys. Status Solidi (b) 43 (1971) 665.

[44] Christian, J. W., The theory of transformations in metals and alloys (Pergamon-Oxford), 1965.

[45] Kaufman, L. and Cohen, M., Progr. Met. Phys. 7 (1958) 165.

[46] Cahn, J., Acta Met. 5 (1957) 169.

[47] Olson, G. B. and Cohen, M., Met. Trans. 7A (1976) 1897.

[48] Raghavan, V. and Cohen, M., Acta Met. 20 (1972) 333, 779. 